\title{
Pseudo interface waves observed at the fluid/porous-medium interface. A comparison of two methods
}

\author{
K. N. van Dalen, ${ }^{\text {a) }}$ G. G. Drijkoningen, and D. M. J. Smeulders \\ Faculty of Civil Engineering and Geosciences, Department of Geotechnology, Delft University of Technology, \\ Stevinweg 1, Delft 2628 CN, The Netherlands
}

(Received 21 June 2010; revised 19 January 2011; accepted 22 January 2011)

\begin{abstract}
At the fluid/porous-medium interface the pseudo-Rayleigh $(p R)$ and pseudo-Stoneley $(p S t)$ waves exist. The relation with the corresponding poles in the slowness plane is not unambiguous, depending on the choice of branch cuts. For a point-force excitation, the far-field Green's functions are computed using vertical branch cuts (method I) implying that the $p R$ - and $p S t$-poles obey the radiation condition. Then, a separate pseudo interface wave is entirely captured by the corresponding pole residue because the loop integral along a branch cut contributes to a body wave only. When hyperbolic branch cuts are used (method II) the poles lie on the "principal" Riemann sheet. Then, also the loop integrals necessarily contribute to the $p R$-wave because the $p R$-pole is different from that in method I. They do not contribute to the $p S t$-wave when the $p S t$-pole lies on the principal Riemann sheet because the pole is identical to that in method I. When the $p S t$-pole has migrated to another Riemann sheet, however, the $p S t$-wave is fully captured by the loop integrals. In conclusion, the phase velocity and attenuation of a separate pseudo interface wave can be computed from the pole location in method I, but should be extracted from the full response in method II.
\end{abstract}

(C) 2011 Acoustical Society of America. [DOI: 10.1121/1.3557040]

PACS number(s): 43.35.Pt, 43.20.Jr, 43.20.Gp, 43.20.Bi [ANN]

Pages: $2912-2922$

\section{INTRODUCTION}

Interface waves that travel along the boundary of a porous medium carry information of the acoustic properties of that medium. They can be utilized to characterize several parameters belonging either to the solid frame or to the saturating fluid. Applications exist in ultrasonic testing of structures, borehole logging in geotechnical and reservoir engineering, and surface seismics in geophysics.

Several theoretical studies were performed on interface waves propagating along the boundary of a porous medium. Most of them were carried out in the context of Biot's theory for wave propagation in fluid-saturated poroelastic solids and discuss the fluid/porous-medium configuration. ${ }^{1-9}$ In general, three types of interface wavemodes can exist at such an interface: The pseudo-Rayleigh $(p R)$ wave, the pseudoStoneley $(p S t)$ wave, and the true interface wave. The former are called "pseudo" because part of their energy is leaking into slower body wavemodes as they propagate along the interface due to their supersonic character. Some conditions for the existence of the pseudo interface waves have been given by Feng and Johnson. ${ }^{2}$ The "true" interface wave is called as such since it is slower than all body wavemodes and therefore non-leaky, and it is comparable to the Stoneley wave at the fluid/elastic-solid interface. It is only present for the closed-pore boundary, where fluid is not free to flow across the interface, or in case of a relatively soft porous frame. ${ }^{2}$

For material characterization using the pseudo interface waves it is important to have predictions of the propagation characteristics of the individual wavemodes, i.e., the velocity

\footnotetext{
a) Author to whom correspondence should be addressed. Electronic mail: k.n.vandalen@tudelft.nl
}

and attenuation. Feng and Johnson ${ }^{2}$ computed phase velocities and attenuations of the pseudo interface waves from the location of the zeroes ( $p R$ - and $p S t$-poles) of the poroelastic Stoneley dispersion equation in the complex plane. Physical constraints for the involved square roots were derived using the radiation condition, defining the Riemann sheets where the zeroes lie on. A similar approach was adopted by Edelman and Wilmanski, ${ }^{4}$ and Gubaidullin et al. ${ }^{7}$

In other papers the full transient response excited by a source is considered, ${ }^{6,9}$ i.e., the acoustic wave motion including all excited wavemodes. The pseudo interface waves are then part of the full response as computed by evaluation of the inverse Fourier integral over horizontal slowness (or wavenumber) using contour integration on the "principal" Riemann sheet. In that case, the residue of a pole contributes to the waveform of a pseudo interface wave, but in a recent paper it has been observed that the waveform can also be affected by the loop integrals along the hyperbolic branch cuts. ${ }^{9}$ Consequently, the wave is not necessarily captured by the residue of the corresponding pole only. Now the question arises why and under which conditions the phase velocity and attenuation of a pseudo interface wave can be computed directly from the pole location.

To answer this question, in this paper we compute the transient point-force response observed at the fluid/porousmedium interface using two different methods to compose the Green's functions (impulse responses). Method I is the representative of Refs. 2, 4, and 7 and method II of Refs. 6 and 9. In method I we use vertical branch cuts for the involved square roots, implying that the contributing poles are formed exactly by the zeroes found using the square-root restrictions of Feng and Johnson. ${ }^{2}$ We show that, in the case of separated waves in the far field, the loop integral along a 
branch cut only contributes to a single body wave. Consequently, the far-field waveform of a separate pseudo interface wave is captured by the residue contribution of the corresponding pole only. In method II we use the hyperbolic branch cuts and we elucidate that the use of these branch cuts is responsible for the contributions of the loop integrals to the pseudo interface waves: There can be various singularities close to the path of integration. Unique physical interpretation is only possible for the $p S t$-pole in a specific situation. Further, we explain the differences and similarities of the poles encountered in the two methods, and elucidate the physical reason of the migration of $p S t$-pole from one to another Riemann sheet, as originally observed in method II. ${ }^{9}$ Numerical examples are shown to illustrate our findings.

We utilize the model describing the three-dimensional transient response due to a point force applied normal to the interface of a fluid/porous-medium configuration (see Fig. 1), ${ }^{9}$ but we restrict ourselves to an observation point on the interface. In Sec. II we summarize the governing equations. We elaborate the two different methods in Sec. III. In Sec. IV we investigate the contributions of poles and branch cuts in more detail. Numerical results are discussed in Sec. V and conclusions are given in Sec. VII.

\section{MODEL}

We consider the configuration of a fluid half-space on top of a fluid-saturated poroelastic half-space, with a point force $F(t)$ applied normal to the interface (see Fig. 1; the schematic response is referred to later). ${ }^{9}$ Both half-spaces are considered to be homogeneous and isotropic, and the interface is an open-pore boundary. In this section we summarize the governing equations.

The behavior in the lower half-space $\left(x_{3}>0\right)$ is governed by the well-known Biot equations of motion for a fluid-saturated poroelastic solid that were extensively

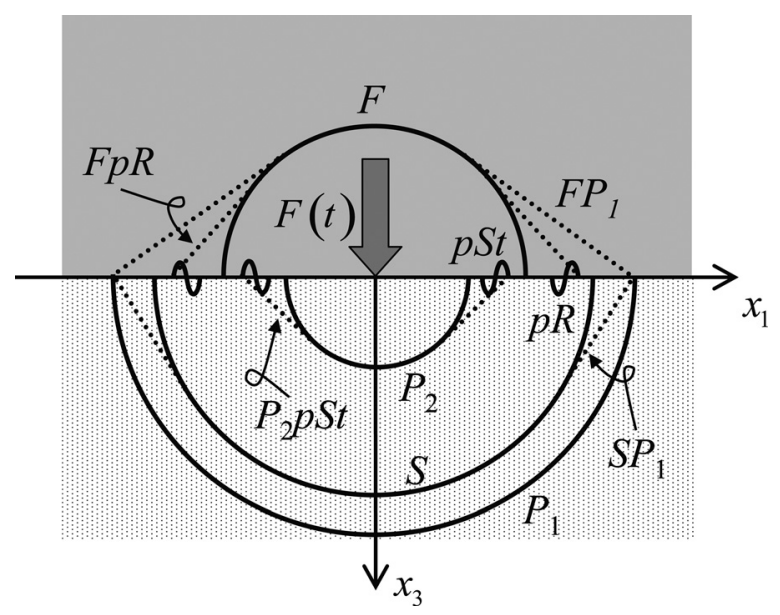

FIG. 1. Point force $F(t)$ applied normal to interface of fluid-saturated porous half-space and fluid half-space, and schematic response (snapshot): fast compressional $\left(P_{1}\right)$ wave, slow compressional $\left(P_{2}\right)$ wave, shear $(S)$ wave, fluid $(F)$ wave, pseudo-Rayleigh $(p R)$ wave and pseudo-Stoneley $(p S t)$ wave. Double-mode symbols (e.g., $\left.S P_{1}\right)$ indicate lateral waves: First symbol denotes wavemode of specific arrival, the second that from which it is radiated. Here, $F$-wave velocity is assumed higher than $P_{2}$-wave velocity and smaller than $S$-wave velocity. For clarity, we omitted arrivals $F S, P_{2} P_{1}, P_{2} S$, $P_{2} p R$, and $P_{2} F$. discussed in this Journal; see e.g., Refs. 10 and 11. We assume that for long-wavelength disturbances with respect to the characteristic pore scale, average local displacements can be defined for the solid (frame) $\mathbf{u}(\mathbf{x}, t)=\left(u_{1}, u_{2}, u_{3}\right)^{T}$ and the fluid $\mathbf{U}(\mathbf{x}, t)=\left(U_{1}, U_{2}, U_{3}\right)^{T}$. The equations of motion are found from usual combination of mass and momentum conservation, and constitutive relations giving ${ }^{10,11}$

$$
\begin{aligned}
\rho_{11} \partial_{t}^{2} \mathbf{u} & +\rho_{12} \partial_{t}^{2} \mathbf{U}+b(t) * \partial_{t}(\mathbf{u}-\mathbf{U}) \\
& =P \nabla \nabla \cdot \mathbf{u}-G \nabla \times \nabla \times \mathbf{u}+Q \nabla \nabla \cdot \mathbf{U} \\
\rho_{12} \partial_{t}^{2} \mathbf{u} & +\rho_{22} \partial_{t}^{2} \mathbf{U}-b(t) * \partial_{t}(\mathbf{u}-\mathbf{U}) \\
& =Q \nabla \nabla \cdot \mathbf{u}+R \nabla \nabla \cdot \mathbf{U}
\end{aligned}
$$

where $\partial_{t}=\partial / \partial t$, the asterisk denotes convolution, and $P, Q$ and $R$ are generalized elastic constants that are related to porosity $\phi$, grain bulk modulus $K_{s}$, fluid bulk modulus $K_{f}$, bulk modulus of porous drained frame $K_{b}$, and shear modulus $G$ of both drained frame and total composite. ${ }^{12}$ The effective densities are defined as

$$
\begin{aligned}
& \rho_{11}=(1-\phi) \rho_{s}-\rho_{12} \\
& \rho_{22}=\phi \rho_{f}-\rho_{12} \\
& \rho_{12}=-\left(\alpha_{\infty}-1\right) \phi \rho_{f}
\end{aligned}
$$

where the tortuosity $\alpha_{\infty} \geq 1$, and hence $\rho_{12} \leq 0$. Solid and fluid densities are denoted as $\rho_{s}$ and $\rho_{f}$, respectively. The linear time-convolution operator $b(t)$ was formulated in the frequency domain as the viscous correction factor by Johnson et al., ${ }^{13}$ according to [cf. Eq. (11)]

$$
\hat{b}(\omega)=b_{0}\left(1+\frac{1}{2} \mathrm{i} M \omega / \omega_{c}\right)^{\frac{1}{2}}
$$

where the viscous damping factor $b_{0}=\phi^{2} \eta / k_{0}$ and $\operatorname{Re}(\hat{b}(\omega)) \geq 0$ for $\omega \geq 0$. Here, $\eta$ denotes the dynamic fluid viscosity and $k_{0}$ the zero-frequency Darcy permeability. The shape factor $M$ is usually taken equal to $1 .{ }^{14}$ The rollover frequency, which represents the transition from low-frequency viscosity-dominated to high-frequency inertia-dominated behavior, is defined as $\omega_{c}=\eta \phi /\left(\alpha_{\infty} \rho_{f} k_{0}\right)$.

The behavior of the upper (fluid) half-space $\left(x_{3}<0\right)$ is governed by the acoustic wave equation

$$
\rho_{F} \partial_{t}^{2} p_{F}=K_{F} \nabla^{2} p_{F}
$$

where $K_{F}$ and $\rho_{F}$ denote the bulk modulus and density of the fluid, respectively, and $p_{F}$ denotes the fluid pressure.

The behavior at the interface is assumed to be governed by conventional open-pore conditions, ${ }^{15}$ i.e., by continuity of volume flux and fluid pressure, and vanishing intergranular vertical stress $\sigma_{33}$ and shear stresses $\sigma_{13}$ and $\sigma_{23} .{ }^{10}$ The open-pore boundary can be a realistic choice to model the fluid/poroelastic-medium interface, ${ }^{16}$ but it is a limiting case of the general situation where a finite surface flow impedance is considered. ${ }^{15}$ When the point force $F(t)$ is applied to the solid, in the limit of $x_{3} \rightarrow 0$ the following conditions should be satisfied, 


$$
\begin{aligned}
& (1-\phi) u_{3}+\phi U_{3}-U_{F, 3}=0, \\
& p_{f}-p_{F}=0, \\
& \sigma_{13}=0, \\
& \sigma_{23}=0, \\
& \sigma_{33}=F(t) \delta\left(x_{1}\right) \delta\left(x_{2}\right),
\end{aligned}
$$

where $\delta(\ldots)$ denotes the Dirac delta function, and $U_{F, 3}$ denotes the vertical particle displacement in the upper half-space.

The medium is considered to be at rest at $t \leq 0$. At infinite distance from the source, the motions are bounded.

\section{GREEN'S FUNCTIONS}

In this section we first summarize the plane-wave domain solution to the set of governing equations [Eqs. (1), (2), and (5)-(10)], as derived earlier. ${ }^{9}$ Then we show how the space-frequency $(\mathbf{x}, \omega)$-domain Green's functions are composed using the two different methods (see Sec. I).

\section{A. Plane-wave domain response}

In order to consider the plane-wave domain response, we apply the Fourier transform over time $t$ according to

$$
\hat{\mathbf{u}}(\mathbf{x}, \omega)=\int_{-\infty}^{\infty} \mathbf{u}(\mathbf{x}, t) \exp (-\mathrm{i} \omega t) d t,
$$

where $\omega$ denotes the angular frequency and i the imaginary unit. Since $\mathbf{u}(\mathbf{x}, t)$ is real-valued it is sufficient to consider $\omega \geq 0$. Following Aki and Richards, ${ }^{17}$ the Fourier transform over horizontal spatial coordinates can be defined as

$\tilde{\mathbf{u}}\left(\mathbf{p}, x_{3}, \omega\right)=\int_{-\infty}^{\infty} \int_{-\infty}^{\infty} \hat{\mathbf{u}}(\mathbf{x}, \omega) \exp (\mathrm{i} \omega \mathbf{p} \cdot \mathbf{r}) d x_{1} d x_{2}$,

where $\mathbf{p}=\left(p_{1}, p_{2}\right)^{T}$ is the horizontal slowness vector and $\mathbf{r}=\left(x_{1}, x_{2}\right)^{T}$ is the horizontal space vector. The transforms are applied similarly to the other field quantities. The hat refers to the $(\mathbf{x}, \omega)$-domain and the tilde to the $\left(\mathbf{p}, x_{3}, \omega\right)$-domain.

The response in the plane-wave domain $\left(\mathbf{p}, x_{3}, \omega\right)$ is described by the physical quantities $\tilde{u}_{i}$ and $\tilde{p}_{f}$ in the lower half-space, grouped in the vector $\tilde{\mathbf{w}}=\left(\tilde{u}_{1}, \tilde{u}_{2}, \tilde{u}_{3},-\phi \tilde{p}_{f}\right)^{T}$, and by $\tilde{p}_{F}$ in the upper half-space. The expressions for the response can be obtained using standard Helmholtz decomposition of the equations of motion, and the boundary conditions. The resulting set of equations can be solved analytically. Finally, the $\left(\mathbf{p}, x_{3}, \omega\right)$-domain solution can be written in terms of Green's functions as

$$
\begin{aligned}
& \tilde{\mathbf{w}}=\tilde{\mathbf{g}}^{+} \hat{F}=\frac{\tilde{\mathbf{n}}^{+}}{\Delta_{S t}} \hat{F}, \\
& \tilde{p}_{F}=\tilde{g}^{-} \hat{F}=\frac{\tilde{n}^{-}}{\Delta_{S t}} \hat{F} .
\end{aligned}
$$

Here, $\tilde{\mathbf{g}}^{+}$and $\tilde{g}^{-}$are the Green's functions for $x_{3}>0$ and $x_{3}<0$, respectively, with numerators $\tilde{\mathbf{n}}^{+}$and $\tilde{n}^{-}$, and $\Delta_{S t}(p, \omega)$ is the "poroelastic Stoneley-wave denominator." $\hat{F}$ is the Fourier transform of the force signature. The Green's functions are linear combinations of the body modes: $\tilde{\mathbf{g}}^{+}$contains the fast compressional $\left(P_{1}\right)$ wave, the slow compressional $\left(P_{2}\right)$ wave, and the vertically-polarized shear $(S)$ wave; $\tilde{g}^{-}$only contains the fluid $(F)$ wave (see Fig. 1). The corresponding propagation terms are $\exp \left(\mp \mathrm{i} \omega q_{\alpha} x_{3}\right)$, where $\alpha=\left\{P_{1}, P_{2}, F, S\right\}$; in the argument, the + relates to the upper medium and the - to the lower. The vertical slownesses are defined as $q_{\alpha}=\left(s_{\alpha}^{2}-p^{2}\right)^{1 / 2}$, with $\operatorname{Im}\left(q_{\alpha}\right) \leq 0$ for real $p$. Here, $s_{\alpha}$ are the body-wave slownesses having $\operatorname{Im}\left(s_{\alpha}\right) \leq 0$ for $\omega \geq 0$, and $p$ denotes the magnitude of the horizontal slowness.

The $(\mathbf{x}, \omega)$-domain Green's functions are found by evaluation of the inverse Fourier transform over horizontal slowness [cf. Eq. (12)]. When cylindrical coordinates are introduced, it follows that ${ }^{9}$

$$
\hat{g}_{3}^{+}=\frac{\omega^{2}}{4 \pi} \int_{-\infty}^{\infty} \frac{\tilde{n}_{3}^{+}}{\Delta_{S t}} H_{0}^{(2)}(\omega p r) p d p
$$

where $r=\left(x_{1}^{2}+x_{2}^{2}\right)^{\frac{1}{2}}$, and $H_{0}^{(2)}(\ldots)$ is the Hankel function of zeroth order and second kind. We only specify the expression for $\hat{g}_{3}^{+}$; the other Green's functions are obtained by simply replacing $\tilde{n}_{3}^{+}$, in Eq. (15) by the other numerators. For brevity, from this point we omit the superscript, i.e., $\hat{g}_{3}^{+}=\hat{g}_{3}$.

Now, we change the real-axis integral into a contour integral in the complex $p$-plane. ${ }^{18}$ The idea is that by integration in the complex plane, contributions from branch cuts and poles can be distinguished. Two different sets of branch cuts are employed to make the square roots single-valued in the entire $p$-plane.

\section{B. Method I: Vertical branch cuts}

In the first method (see also Refs. 2, 4, and 7) we compose the Green's functions using branch cuts for the square roots $q_{\alpha}$ along the vertical half-lines departing from the corresponding branch points being the body-wave slownesses $s_{\alpha}$, as shown in Fig. 2. In this figure also the closed

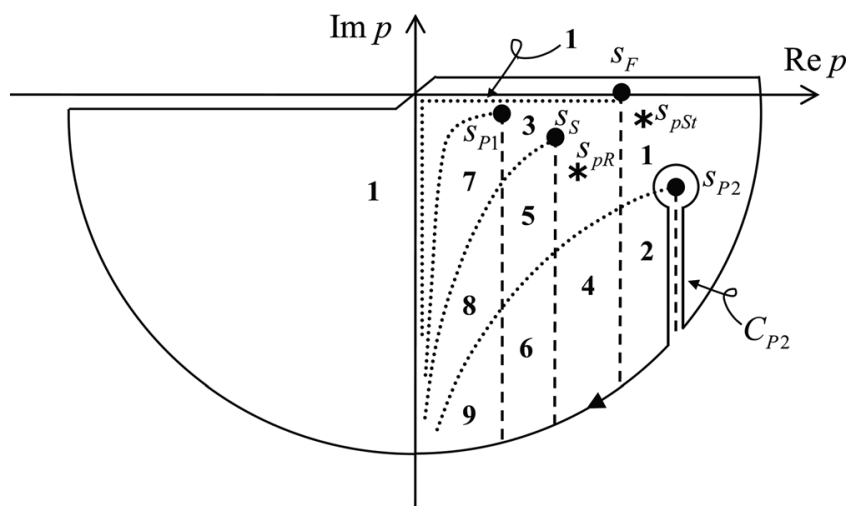

FIG. 2. Complex $p$-plane for method I with (- $)$ vertical branch cuts of square roots $q_{\alpha}$ departing from corresponding $(\bullet)$ branch points $s_{\alpha}, \alpha=\left\{P_{1}\right.$, $\left.P_{2}, F, S\right\}$. Poles $(*) s_{p R}$ (pseudo-Rayleigh) and $\mathbf{s}_{p S t}$ (pseudo-Stoneley) are zeroes of poroelastic Stoneley denominator; see Eq. (18). Only part of the closed integration contour (-) is displayed: Real axis, arc in lower half-plane and loop $C_{P 2}$ along $q_{P 2}$-branch cut. Direction of integration is indicated. Every (...) line indicates transition between two Riemann sheets as indicated by numbers (Fig. 3 and Table I). Here, $F$-wave velocity is assumed higher than $P_{2}$-wave velocity and smaller than $S$-wave velocity. 
integration contour is displayed, which is formed by the entire real axis, the loops along the branch cuts and around the branch points, and an arc of infinite radius in the lower half-plane. For $\operatorname{Re}(p) \leq 0$ the horizontal part of the contour lies just below the axis due to the presence of a branch cut of the Hankel function at the negative real axis. ${ }^{19}$

The meaning of the dotted hyperbolas in Fig. 2 is illustrated in Fig. 3, where the behavior of $q_{P 1}$ in the complex plane is sketched. There is a (abrupt) sign change over the vertical branch cut and throughout the rest of the $p$-plane the magnitude changes smoothly. Along the dotted hyperbolas either the imaginary part [for $\operatorname{Re}(p)<\operatorname{Re}\left(s_{P 1}\right)$ ], or the real part [for $\operatorname{Re}(p)>\operatorname{Re}\left(s_{P 1}\right)$ ], is zero. ${ }^{20}$ The other roots behave similarly. Hence, the dotted lines in Fig. 2 indicate where $\operatorname{Im}\left(q_{\alpha}\right)=0$. Crossing such a line means that one moves from one to another Riemann sheet.

Consequently, inside the closed contour (Fig. 2) nine different Riemann sheets can be distinguished. Throughout the entire complex plane the signs of the square roots $q_{\alpha}$ are calculated given the requirement that $\operatorname{Im}\left(q_{\alpha}\right) \leq 0$ on the real $p$-axis, which is the original path of integration [see Eq. (15)]. ${ }^{17}$ We indicate every Riemann sheet according to the sign of $\operatorname{Im}\left(q_{\alpha}\right)$, e.g., $\operatorname{sgn}\left(\operatorname{Im}\left(q_{P 1}, q_{P 2}, q_{F}, q_{S}\right)\right)=(----)$ is the sheet where all roots have imaginary parts smaller than or equal to zero, which is the "principal" Riemann sheet. ${ }^{17,21}$ In Fig. 2 this sheet is indicated by 1 . In Table I an overview is given of all Riemann sheets inside the contour.

\section{Method II: Hyperbolic branch cuts}

In the second method (see also Refs. 6 and 9) we compose the Green's functions using branch cuts exactly along the hyperbolic lines $\operatorname{Im}\left(q_{\alpha}\right)=0$; see Fig. 4. This implies that $\operatorname{Im}\left(q_{\alpha}\right)<0$ over the rest of $p$-plane, ${ }^{20}$ and ensures the decay of the exponential terms $\exp \left(\mp \mathrm{i} \omega q_{\alpha} x_{3}\right)$ for large $|p|$ [present in Eqs. (13) and (14)]. The $q_{F}$-branch cut coincides with the imaginary axis and part of the real axis since the slowness of the fluid $\left(s_{F}\right)$ is real-valued. The current branch cuts imply that the integration is performed entirely on the principal Riemann sheet.

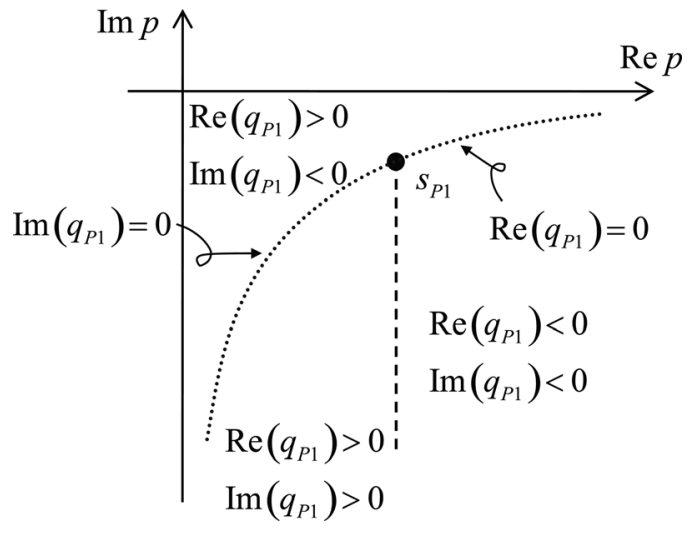

FIG. 3. Complex $p$-plane in method I with (--) $q_{P 1}$-branch cut departing from branch point $s_{P 1}$. Hyperbolic lines (..) indicate where either $\operatorname{Re}\left(q_{P 1}\right)=0$ or $\operatorname{Im}\left(q_{P 1}\right)=0$. In various areas signs of real and imaginary parts of $q_{P 1}$ are indicated.
TABLE I. Various Riemann sheets present inside closed contours in Figs. 2 and 4. Every Riemann sheet is characterized by signs of imaginary parts of involved square roots.

\begin{tabular}{lcccc}
\hline \hline & $\operatorname{Im}\left(q_{P 1}\right)$ & $\operatorname{Im}\left(q_{P 2}\right)$ & $\operatorname{Im}\left(q_{F}\right)$ & $\operatorname{Im}\left(q_{S}\right)$ \\
\hline 1 & - & - & - & - \\
2 & - & + & - & - \\
3 & - & - & + & - \\
4 & - & + & + & - \\
5 & - & - & + & + \\
6 & - & + & + & + \\
7 & + & - & + & - \\
8 & + & - & + & + \\
9 & + & + & + & + \\
\hline \hline
\end{tabular}

In Fig. 4 also the closed contour is displayed, similar as in Fig. 2.

\section{Inverse Fourier transform}

We now compose the $(\mathbf{x}, \omega)$-domain Green's function $\hat{g}_{3}$ as a summation of the contributions of the branch cuts and the residues of the poles that lie inside the contour. In both methods Cauchy's residue theorem ${ }^{18}$ leads to

$$
\hat{g}_{3}=\sum_{\alpha} \hat{g}_{3, \alpha}+\sum_{\beta} \hat{g}_{3, \beta},
$$

where $\hat{g}_{3, \alpha}$ denotes the contribution of the loop integral along the $q_{\alpha}$-branch cut,

$$
\hat{g}_{3, \alpha}=-\frac{\omega^{2}}{4 \pi} \int_{C_{\alpha}} \frac{\tilde{n}_{3}}{\Delta_{S t}} H_{0}^{(2)}(\omega p r) p d p
$$

and $\alpha=\left\{P_{1}, P_{2}, F, S\right\}$. Here, $C_{\alpha}$ represents the integration loop. In Eq. (16), $\hat{g}_{3, \beta}$ denotes the residue contribution of a first-order pole $s_{\beta}$ of the integrand inside the integration contour, which is typically the pseudo-Rayleigh $(p R)$ pole or the pseudo-Stoneley ( $p S t)$ pole (see Figs. 2 and 4$){ }^{9}$

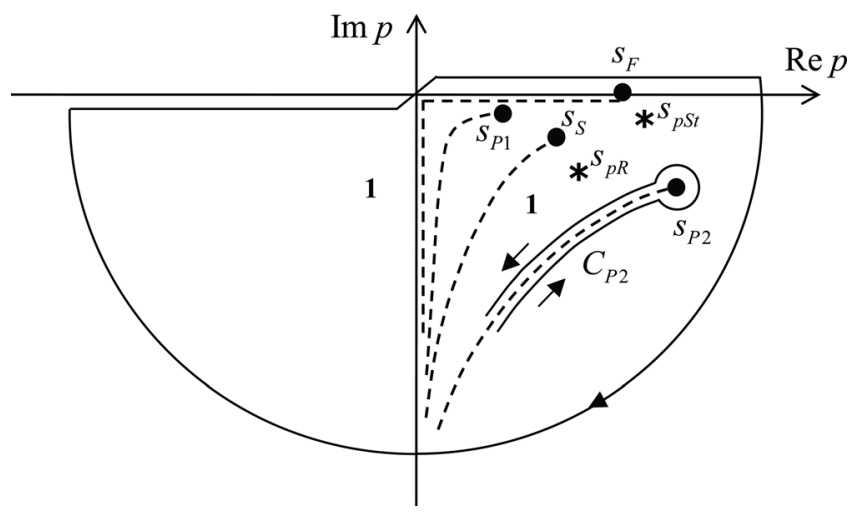

FIG. 4. Complex $p$-plane for method II with (- -) hyperbolic branch cuts of square roots $q_{\alpha}$ departing from corresponding $(\bullet)$ branch points $s_{\alpha}$, $\alpha=\left\{P_{1}, P_{2}, F, S\right\}$. Poles (*) $s_{p R}$ (pseudo-Rayleigh) and $s_{p S t}$ (pseudo-Stoneley) are zeroes of poroelastic Stoneley denominator; see Eq. (18). Only part of the closed integration contour (-) is displayed (cf. Fig. 2). Direction of integration is indicated. Integration is done entirely on the principal Riemann sheet (indicated by 1; Table I). Here, $F$-wave velocity is assumed higher than $P_{2}$-wave velocity and smaller than $S$-wave velocity. 


$$
\hat{g}_{3, \beta}=-2 \pi \mathrm{i}\left[\frac{\omega^{2}}{4 \pi} \frac{\tilde{n}_{3}}{\partial_{p} \Delta_{S t}} H_{0}^{(2)}(\omega p r) p\right]_{p=s_{\beta}} .
$$

Obviously, both methods give the same Green's function $\hat{g}_{3}$, but due to the different branch cuts the integrands are defined differently (we consider $\tilde{n}_{3} / \partial_{p} \Delta_{S t}$ on different Riemann sheets) and consequently, the contributions of the loop integrals $\hat{g}_{3, \alpha}$ and of the pole residues $\hat{g}_{3, \beta}$ are not the same in both methods. We can even encounter different poles on the different Riemann sheets on which the integration is performed.

\section{PHYSICAL INTERPRETATION OF SEPARATE CONTRIBUTIONS OF THE FAR-FIELD INTERFACE RESPONSE}

In this section we determine to what wavemode(s) of the interface response the loop integral along a single branch cut and a single pole residue contribute, respectively. To facilitate this we only consider the far-field situation and we approximate the loop integral using the saddle-point method. This leads to expressions that have a straightforward physical interpretation. We only elaborate the separate contributions for method I because for method II it is clear beforehand that the loop integral along a branch cut can contribute to several waves in the full response, including the pseudo interface waves. ${ }^{9}$ Unique physical interpretation is only possible for the pSt-pole in a specific situation, as we will discuss in Sec. V.

\section{A. Approximate pole contributions}

First, we consider the residue contributions $\hat{g}_{3, \beta}^{I}\left(x_{3}=0\right)$ as given by Eq. (18), where the superscript "I" refers to method I. Considering the wavefield in the far field $(|\omega p r| \gg 1)$, we can write ${ }^{19}$

$$
\begin{aligned}
\hat{g}_{3, \beta}^{I}\left(x_{3}=0\right) & \left.\cong\left(\frac{\omega^{3}}{2 \pi r}\right)^{\frac{1}{2}} \frac{\tilde{n}_{3}}{\partial_{p} \Delta_{S t}}\right|_{x_{3}=0 ; p=s_{\beta}} s_{\beta}^{\frac{1}{2}} \mathrm{e}^{-\mathrm{i} \omega s_{\beta} r-\mathrm{i} \frac{\pi}{4}} \\
& =A_{\beta}(\omega, r) e^{-\mathrm{i} \omega \operatorname{Re}\left(s_{\beta}\right) r+\mathrm{i} \varphi_{\beta}(\omega)}
\end{aligned}
$$

where $\operatorname{Re}\left(s_{\beta}^{\frac{1}{2}}\right)>0$, and $A_{\beta}$ is the amplitude and $\varphi_{\beta}$ is the $r$ independent phase angle of the wavemode that has phase propagation slowness $\operatorname{Re}\left(s_{\beta}\right)$, i.e., the pseudo-Rayleigh $(p R)$ or the pseudo-Stoneley $(p S t)$ wave. All contributing poles $s_{\beta}$ have $\operatorname{Im}\left(s_{\beta}\right)<0$ as they lie inside the integration contour. This ensures the vanishing of the displacements for $r \rightarrow \infty$ [Eq. (19)].

\section{B. Approximate branch-cut contributions}

Next, we focus on the contributions of the branch cuts $\hat{g}_{3, \alpha}^{I}\left(x_{3}=0\right)$ as given by Eq. (17). Introducing $p=s_{\alpha}-\mathrm{i} \xi$, where $\xi$ is a local variable of integration and considering the far field $(|\omega p r| \gg 1)$, we can write for the contribution of $C_{\alpha}$-taking both sides ( $L$ : left; $R$ : right) in one integral,

$$
\begin{aligned}
\hat{g}_{3, \alpha}^{I}\left(x_{3}=0\right) \cong & \left(\frac{\omega^{3}}{8 \pi^{3} r}\right)^{\frac{1}{2}} \mathrm{e}^{-\mathrm{i} \omega s_{\alpha} r+\mathrm{i} \frac{3}{4} \pi} \\
& \times \int_{0}^{\infty}\left(\frac{\tilde{n}_{3, L}}{\Delta_{S t, L}}-\frac{\tilde{n}_{3, R}}{\Delta_{S t, R}}\right)_{x_{3}=0} p^{\frac{1}{2}} \mathrm{e}^{-\omega r \xi} d \xi \\
= & A_{\alpha}(\omega, r) \mathrm{e}^{-\mathrm{i} \omega \operatorname{Re}\left(s_{\alpha}\right) r+\mathrm{i} \varphi_{\alpha}(\omega, r)}
\end{aligned}
$$

where the signs of all roots $q_{\alpha}$ can be determined from Figs. 2 and 3 , and Table I, and where $\operatorname{Re}\left(p^{\frac{1}{2}}\right) \geq 0 \forall p ; ;^{17} A_{\alpha}$ is some amplitude and $\varphi_{\alpha}$ a phase angle. We now show how the integral over $\xi$ contributes to $A_{\alpha}$ and $\varphi_{\alpha}$ using a saddle-point approximation. We only show the approximation of the loop integral $C_{F}$, but the others $\left(C_{P 1}, C_{P 2}\right.$, and $\left.C_{S}\right)$ can be approximated similarly.

From Eq. (21) it is observed that the integration path is the path of steepest descent of the far-field integrand at $x_{3}=0 .{ }^{22}$ The saddle point coincides with the branch point of the particular branch cut, i.e., $p=s_{\alpha}$. This is a limiting case of the more general situation where $x_{3} \neq 0$ and the saddle point lies at $p=s_{\alpha} r / R_{0}$, where $R_{0}=\left(x_{1}^{2}+x_{2}^{2}+x_{3}^{2}\right)^{\frac{1}{2}} \cdot{ }^{17,23,24}$

We employ Watson's lemma ${ }^{25}$ to evaluate the integral for $\omega r \rightarrow \infty$

$$
\begin{aligned}
\int_{0}^{\infty} \xi^{+a} f_{F}(\xi, \omega) e^{-\omega r \xi} d \xi= & \left.\sum_{n=0}^{\infty} \partial_{\xi}^{n} f_{F}(\xi, \omega)\right|_{\xi=0} \\
& \times \frac{\Gamma(a+n+1)}{(\omega r)^{a+n+1}}
\end{aligned}
$$

where $\partial_{\xi}^{n}=\partial^{n} / \partial \xi^{n}, a>-1, \Gamma(\ldots)$ is the gamma function, and

$$
f_{F}(\xi, \omega)=\xi^{-a} p^{\frac{1}{2}}\left(\frac{\tilde{n}_{3, L}}{\Delta_{S t, L}}-\frac{\tilde{n}_{3, R}}{\Delta_{S t, R}}\right)_{x_{3}=0}
$$

For the situation that $\operatorname{Re}\left(s_{P 2}\right)>s_{F}$ (which is the case in the numerical examples that we will consider), we have along the integration path (see Figs. 2 and 3, and Table I)

$$
\begin{aligned}
& p=s_{F}-\mathrm{i} \xi \\
& \operatorname{Im}\left(q_{P 1}, q_{S}\right)<0, \\
& \operatorname{Re}\left(q_{P 2}\right)>0, \\
& \operatorname{Im}\left(q_{F, L}\right) \geq 0, \quad \operatorname{Im}\left(q_{F, R}\right) \leq 0 .
\end{aligned}
$$

For $a=\frac{1}{2}, \partial_{\xi}^{n} f_{F}(\xi, \omega)$ is finite at $\xi=0$ (which can be shown using MAPLE ${ }^{\mathbb{C}}$ ) and hence, using $\Gamma\left(\frac{3}{2}\right)=\frac{1}{2} \sqrt{\pi}$ Eq. (21) can be approximated as

$$
\hat{g}_{3, F}^{I, o s}\left(x_{3}=0\right)=\frac{1}{4 \sqrt{2} \pi r^{2}} f_{F}(0, \omega) \mathrm{e}^{-\mathrm{i} \omega S_{F} r+\mathrm{i} \frac{3}{4} \pi},
$$

which is the "ordinary" saddle-point approximation ${ }^{22}$ (indicated by superscript "os") because it only uses the zerothorder term [see Eq. (23)]. The error is of $\mathcal{O}\left(\omega^{-1} r^{-3}\right)$ provided that $f_{F}(0, \omega)$ is not singular in the vicinity of the saddle point.

A pole lying in the vicinity of the saddle point invalidates the ordinary approximation. For instance, as we will discuss in a numerical example (Sec. V), the $p S t$-pole can lie very close to the fluid-wave branch point, strongly affecting the behavior of $f_{F}(\xi, \omega)$. We now show how to include the effect of the proximity of $p S t$-pole in the approximation of the fluid branch-cut contribution. We use the "modified" saddle-point method where the singular part of the integrand is split off and handled separately. ${ }^{22,23,26}$ 
First, we make a transform of variable, $\xi=\zeta^{2} /(\omega r)$, so that

$$
p=s_{F}-\mathrm{i} \zeta^{2} /(\omega r),
$$

and

$$
\hat{g}_{3, F}^{I}\left(x_{3}=0\right) \cong\left(\frac{\omega}{8 \pi^{3} r^{3}}\right)^{\frac{1}{2}} \mathrm{e}^{-\mathrm{i} \omega S_{F} r+\mathrm{i}_{\frac{3}{4}} \pi} I,
$$

where

$$
\begin{aligned}
& I=\int_{0}^{\infty} y(p, \omega) \mathrm{e}^{-\zeta^{2}} d \zeta \\
& y(p, \omega)=2 \zeta p^{\frac{1}{2}}\left(\frac{\tilde{n}_{3, L}}{\Delta_{S t, L}}-\frac{\tilde{n}_{3, R}}{\Delta_{S t, R}}\right)_{x_{3}=0} .
\end{aligned}
$$

We split off the singular part from $y(p, \omega)$ by defining

$$
y(p, \omega)=w(p, \omega)+\frac{y_{p S t}}{p-s_{p S t}},
$$

where $y_{p S t}=y_{p S t}(\omega)$ according to

$$
\begin{aligned}
y_{p S t} & =\lim _{p \rightarrow s_{p S t}}\left(\left(p-s_{p S t}\right) y(p, \omega)\right), \\
& =-\left.2 \zeta_{p S t} s_{p S t}^{\frac{1}{2}} \frac{\tilde{n}_{3, R}}{\partial_{p} \Delta_{S t, R}}\right|_{x_{3}=0 ; p=s_{p S t}} .
\end{aligned}
$$

At $p=s_{p S t}$ the roots take on the same signs as at the right side of the $q_{F}$-branch cut; see Eqs. (26)-(28). The quantity $\zeta_{p S t}$ is the value that $\zeta$ assumes at the pSt-pole; from Eq. (30) we find

$$
\zeta_{p S t}=\left(\mathrm{i} \omega r\left(s_{p S t}-s_{F}\right)\right)^{\frac{1}{2}}
$$

where $\operatorname{Im}\left(\zeta_{p S t}\right)<0$. It represents a normalized distance between the fluid-wave branch point (saddle point) and the pSt-pole. Its magnitude squared is the so-called "numerical distance." Combining Eqs. (32) and (34), we have

$$
\begin{aligned}
& I=I_{1}+I_{2}, \\
& I_{1}=\int_{0}^{\infty} w(p, \omega) \mathrm{e}^{-\zeta^{2}} d \zeta, \\
& I_{2}=\int_{0}^{\infty} \frac{y_{p S t}}{p-s_{p S t}} \mathrm{e}^{-\zeta^{2}} d \zeta .
\end{aligned}
$$

Watson's lemma can now again be applied to approximate $I_{1}$ since the nearby singularity has been removed. The approximation of the first term of $I_{1}[y(p, \omega)]$ leads to the result of Eq. (29). To approximate the second term of $I_{1}\left[-y_{p S t} /\right.$ $\left.\left(p-s_{p S t}\right)\right]$ we need to include two terms of Watson's lemma to get the same order in $\omega r$. The integral $I_{2}$ can be expressed in terms of the complementary error function. ${ }^{27}$ The final result of the modified saddle-point approximation (indicated by superscript "ms") is found by combining Eqs. (31) and (38)-(40)

$$
\begin{aligned}
\hat{g}_{3, F}^{I, m s}\left(x_{3}=0\right)= & \frac{1}{4 \sqrt{2} \pi r^{2}} \mathrm{e}^{-\mathrm{i} \omega S_{F} r+\mathrm{i} \frac{3}{4} \pi} \\
& \times\left(f_{F}(0, \omega)+\frac{(\omega r)^{\frac{1}{2}} y_{p S t}}{s_{p S t}-s_{F}}\left(1+\frac{1}{2 \zeta_{p S t}^{2}}\right)\right. \\
& \left.+\sqrt{\pi} \frac{(\omega r)^{\frac{3}{2}} y_{p S t}}{\zeta_{p S t}} \operatorname{erfc}\left(\mathrm{i} \zeta_{p S t}\right) \mathrm{e}^{-\zeta_{p S t}^{2}}\right) .
\end{aligned}
$$

The second and third terms inside the parenthesis are the correction terms of the modified saddle-point approximation with respect to the ordinary one; they cancel when $\left|\zeta_{p S t}\right| \rightarrow$ $\infty$, i.e., when the pole is far away from the saddle point.

In general, also poles that do not lie inside the closed contour can influence the integrands of the loop integrals along the branch cuts. For instance, the $\bar{P}$-poles that are located close to $p=s_{P 1},{ }^{9}$ can lie on sheet 1 and the $(+--$ -)-sheet, respectively, below sheets 3 and 7 (see Fig. 2), and still invalidate the ordinary saddle-point approximation. ${ }^{22}$ In practice, it is probably the best to evaluate the full integrals numerically, which ensures that all effects of nearby poles are included properly. However, the current approximations allow us to physically interpret the separate contributions in method I.

\section{Physical interpretation of contributions}

Using the results of the previous sections (Secs. IV A and IV B) we are able to physically interpret the separate contributions of the Green's functions. Comparing Eqs. (21), (22) and (29), we observe that in the case where there is no pole in the vicinity of the saddle point, the integral over $\xi$ contributes to the phase angle $\varphi_{F}$ such that it is only $\omega$-dependent: $\varphi_{F}=\varphi_{F}(\omega)$ (this is also the case if more terms of Watson's lemma are included). It does not modify the phase slowness of the exponential term. Therefore, in the far field, a branch-cut contribution in method I only corresponds to a separate body wave in the interface response. Its geometrical decay behaves as $r^{-2}$, which is typical for a body/head wave propagating along the interface. ${ }^{17}$ Consequently, if the loop integral only contributes to a separate body wavemode, the far-field residue contribution of a pole [ $p R$ or $p S t$; Eqs. (19) and (20)] must capture the entire waveform of a separate pseudo interface wave in the interface response, i.e., the $p R$ or the $p S t$-wave. The geometrical decay of the pole residue behaves as $r^{-\frac{1}{2}}$, which is typical for an interface wave.

For the case where there is a pole close to the saddle point, we observe that the integral over $\xi$ contributes to the phase angle $\varphi_{F}$ such that it is also $r$-dependent: $\varphi_{F}=\varphi_{F}(\omega$, r) [cf. Eqs. (21), (22), and (41)]. In Eq. (41) the term $\operatorname{erfc}\left(\mathrm{i} \zeta_{p S t}\right) \exp \left(-\zeta_{p S t}^{2}\right)$ seemingly describes some dispersion of the $F$-wave. However, this term is merely a consequence of the interference of the body wave and the interface wave due to the proximity of the pole and the saddle point. In this case, separate interface and body waves cannot be distinguished (see also Sec V).

In method II the branch cuts follow curved paths in the complex plane. Hence, the integrand of a loop integral can encounter several singularities in the complex plane and thus 
contribute to the waveforms of the corresponding wavemodes. This is the reason that in method II, a body wave cannot be attributed to a single branch-cut contribution and, as a consequence, the residue contribution of an individual pole does not necessarily contain the entire waveform of the corresponding pseudo interface wave.

\section{NUMERICAL RESULTS}

In this section, we subsequently discuss various wavemodes that are excited by the point force and verify to which part (poles and branch cuts) of the Green's functions their waveforms are related, in both the methods I and II. We show the transient interface responses for three different fluid/porous-medium configurations (see Table II) yielding illustrative results for the theoretical elaborations. For clarity, we adhere to the distinction between separate and interfering wavemodes (Secs. V A and V B, respectively) as introduced Sec. IV C. Finally, in Sec. V C, we address the migration of poles, which we first encounter in Sec. V A.

In the first and third configurations the porous medium is a water-saturated Bentheimer sandstone. ${ }^{9}$ The upper halfspace is either a light fluid or water. The second configuration is identical to that of Feng and Johnson. ${ }^{2,3}$ For every configuration, we show the vertical component of particle velocity $v_{3}$ for an observation point at the interface $x_{3}=0$ and offset $r=x_{1}=0.10 \mathrm{~m}$. The point force has Ricker signature, ${ }^{28}$

$$
F(t)=F_{\max }\left(\frac{1}{2} \omega_{0}^{2} \bar{t}^{2}-1\right) \exp \left(-\frac{1}{4} \omega_{0}^{2} \bar{t}^{2}\right),
$$

where $\bar{t}=t-t_{s}(t>0), \omega_{0}=2 \pi f_{0}$ and the center frequency $f_{0}=500 \mathrm{kHz}$ (see Fig. 5). The magnitude $F_{\max }=2.5 \cdot 10^{-7}$ $\mathrm{N}$ and time shift $t_{s}=5 \mu \mathrm{s}$. We have performed the integration over $p$ for the frequency range $0<f \leq 2 \mathrm{MHz}$. The particle velocity is obtained from multiplication of the spectra of the Green's function by the source signature [Eq. (13)], and by $\mathrm{i} \omega$. A standard fast Fourier-transform algorithm is applied for the transform to the time domain.

For both methods we have evaluated the loop integrals by numerical integration of the exact integrands [Eq. (17); no approximations involved]. Similarly, in the evaluation of

TABLE II. Various configurations for which the transient response is considered. The type of sandstone is Bentheimer. ${ }^{9}$ For fused glass beads, ${ }^{2}$ bulk modulus of drained matrix is chosen as $K_{b}=10 \mathrm{GPa}$ and permeability as $k_{0}=10 \mu \mathrm{m}^{2}$. Saturating fluid (water) has bulk modulus $K_{f}=2.22 \mathrm{GPa}$ and density $\rho_{f}=1000 \mathrm{kgm}^{-3}$. Upper half-space is filled with either light fluid $\left(K_{F}=\frac{1}{10} K_{f}, \rho_{F}=\frac{1}{8} \rho_{f}\right)$ or water $\left(K_{F}=K_{f}, \rho_{F}=\rho_{f}\right)$. For every configuration the poles [pseudo-Stoneley $(p S t)$ and pseudo-Rayleigh $(p R)$ ] present inside integration contour are indicated for both methods (I, II).

\begin{tabular}{llllll}
\hline \hline Porous solid & $\begin{array}{l}\text { Saturating } \\
\text { fluid }\end{array}$ & $\begin{array}{l}\text { Upper } \\
\text { half-space }\end{array}$ & $\begin{array}{l}\text { Poles } \\
\text { method I }\end{array}$ & $\begin{array}{l}\text { Poles } \\
\text { method II }\end{array}$ \\
\hline 1 & Sandstone & Water & Light fluid & $p R, p S t$ & $p R, p S t$ \\
2 & Glass beads & Water & Water & $p R, p S t$ & $p S t^{\mathrm{a}}$ \\
3 & Sandstone & Water & Water & $p R,{ }^{\mathrm{b}} p S t$ & $p S t$ \\
\hline \hline
\end{tabular}

${ }^{\text {a }}$ Not present inside integration contour for $f \geq 310 \mathrm{kHz}$.

${ }^{\mathrm{b}}$ Its residue is not shown.
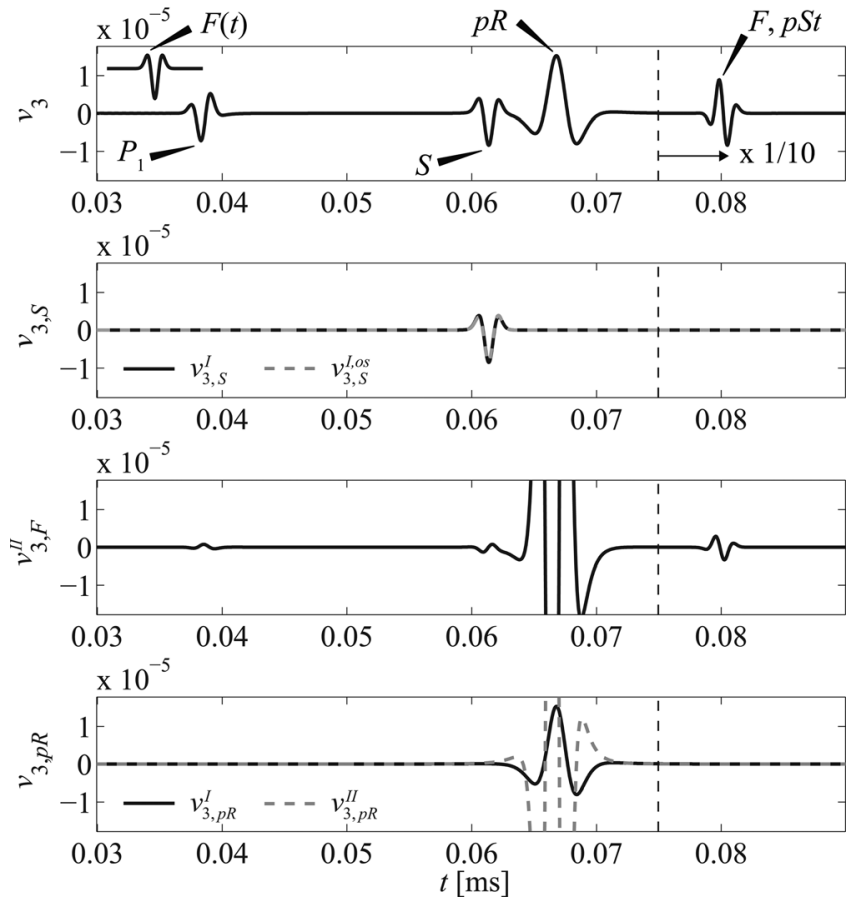

FIG. 5. Bentheimer/light-fluid (configuration 1): Full response $\left(v_{3}\right)$ including source signature $F(t)$ [Eq. (42)], $q_{S}$-branch cut contribution in method I $\left(v_{3, S}^{I}\right)$ and its approximation $\left(v_{3, S}^{I, O S}\right), q_{F}$-branch cut contribution $\left(v_{3, F}^{I I}\right)$ and $p R$-pole residues $\left(v_{3, p R}^{I}, v_{3, p R}^{I I}\right)$, at $x_{2}=x_{3}=0$ and $x_{1}=0.10 \mathrm{~m}$. Residue $v_{3, p R}^{I I}$ and $p R$-wave part of $v_{3, F}^{I I}$ are not entirely visible on scale of this figure. Units are $\left[\mathrm{ms}^{-1}\right]$. From dashed vertical line onwards, responses have been scaled by $\frac{1}{10}$.

the pole residues we have used the full expressions [Eq. (18)]. The poles inside the closed integration contour contributing a residue are given in Table II. We have identified the pseudo-Stoneley $(p S t)$ and the pseudo-Rayleigh $(p R)$ poles by applying the Principle of the Argument to the Stoneley denominator $\Delta_{S t}$ (this principle gives the number of poles inside a closed integration contour). ${ }^{9,18}$ In method I the $p S t$-pole lies on sheet 1 or 2 , and the $p R$-pole on sheet 3 or 4 (see Fig. 2 and Table I). In method I the poles all lie on sheet 1 (see Fig. 4).

For all three configurations the full responses and separate contributions are displayed in Fig. 5 and 7-9. Obviously, the full responses are the same in both methods (and not displayed separately). We have identified the different arrivals

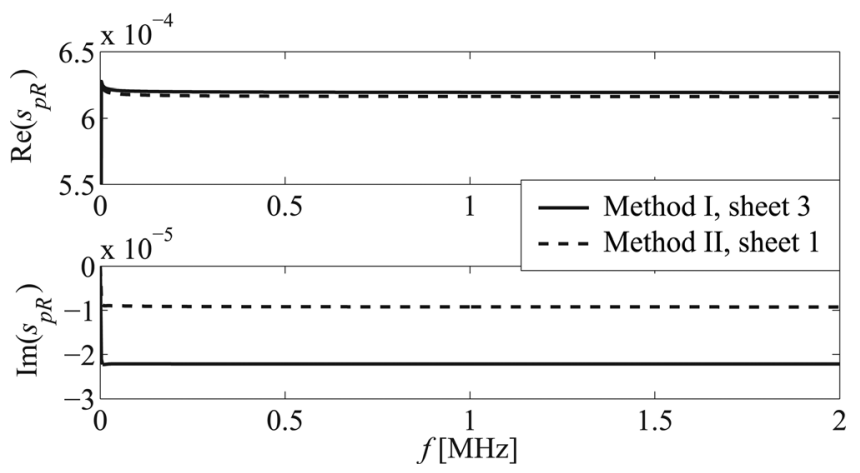

FIG. 6. Bentheimer/light fluid (configuration 1): Location of $p R$-pole in methods I and II. Units are $\left[\mathrm{ms}^{-1}\right]$. 

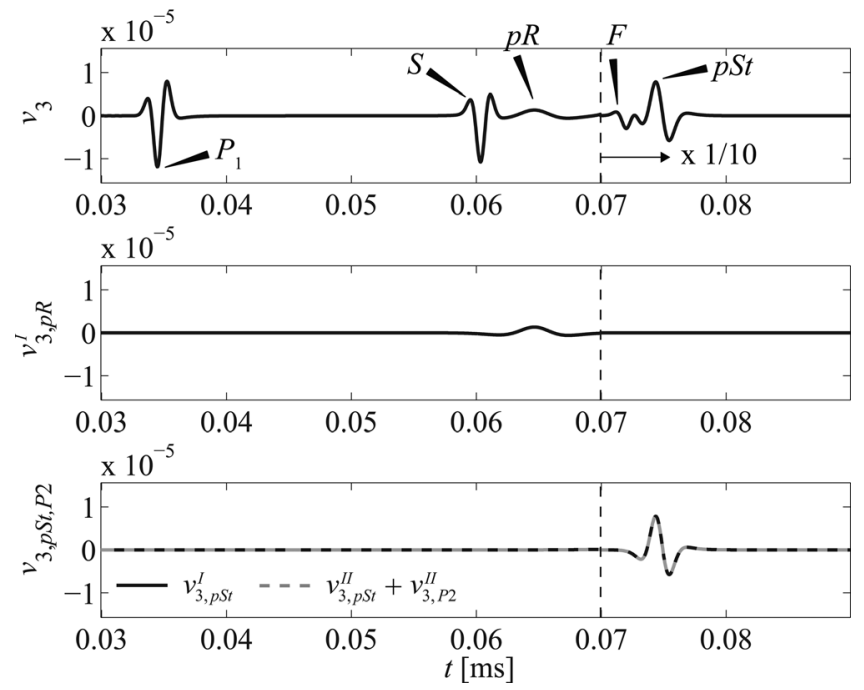

FIG. 7. Glass beads/water (configuration 2): Full response $\left(v_{3}\right), p R$-pole residue $\left(v_{3, P R}^{I}\right)$ and $p S t$-wave as constituted by either $p S t$-pole residue $\left(v_{3, p S t}^{I I}\right)$ and $q_{P 2}$-branch cut contribution $\left(v_{3, P 2}^{I I}\right)$, or only pSt-pole residue $\left(v_{3, p S t}^{I}\right)$, at $x_{2}=x_{3}=0$, and $x_{1}=0.10 \mathrm{~m}$. Units are $\left[\mathrm{ms}^{-1}\right]$. From dashed vertical line onwards, responses have been scaled by $\frac{1}{10}$.

in the full responses using the phase velocities as obtained from the modal slownesses. For the sake of clarity, a schematic snapshot of the full response with the different arrivals is shown in Fig. 1. Here, body waves are indicated by the solid lines and interface waves by short wavelets; lateral waves (either head waves or wavefronts radiated by the pseudo interface waves) are indicated by dotted lines. At the interface, head waves and body waves coincide and therefore, the head waves are not indicated separately in the figures in this section. Superscripts (I and II) refer to the different methods.

\section{A. Separate wavemodes}

We consider the separate (non-interfering) wavemodes present in the full responses. First, we consider the $S$-wave. It can be observed that, in the response of configuration 1 (Fig. 5), its waveform is fully captured by the contribution of the $q_{S}$-branch cut $v_{3, S}^{I}$ in method I [Eq. (17)]. The ordinary saddle-point approximation $v_{3 . S}^{I, o S}$ [Eq. (29)], which is also shown, provides a good approximation of the exact result. In
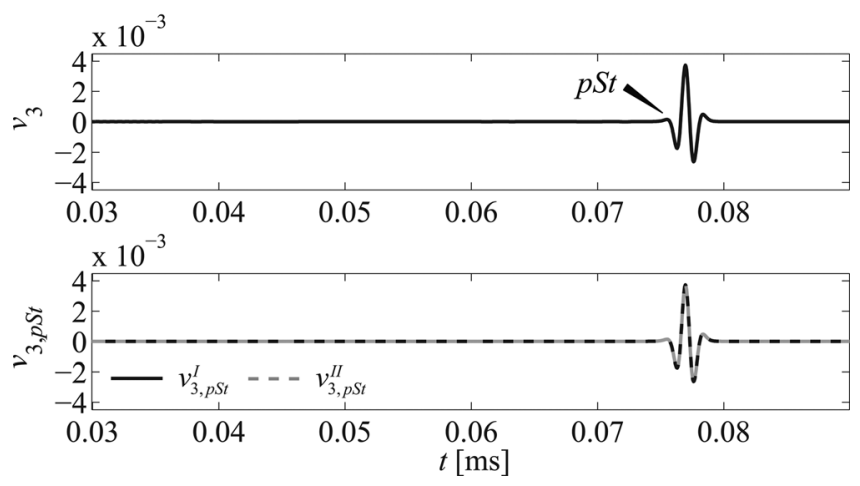

FIG. 8. Bentheimer/water (configuration 3): Full response $\left(v_{3}\right)$ and $p S t$-pole residues $\left(v_{3, p S t}^{I}, v_{3, p S t}^{I I}\right)$, at $x_{2}=x_{3}=0$, and $x_{1}=0.10 \mathrm{~m}$. In $v_{3}$, only the $p S t$ wave can be observed on the scale of this figure. Units are $\left[\mathrm{ms}^{-1}\right]$.
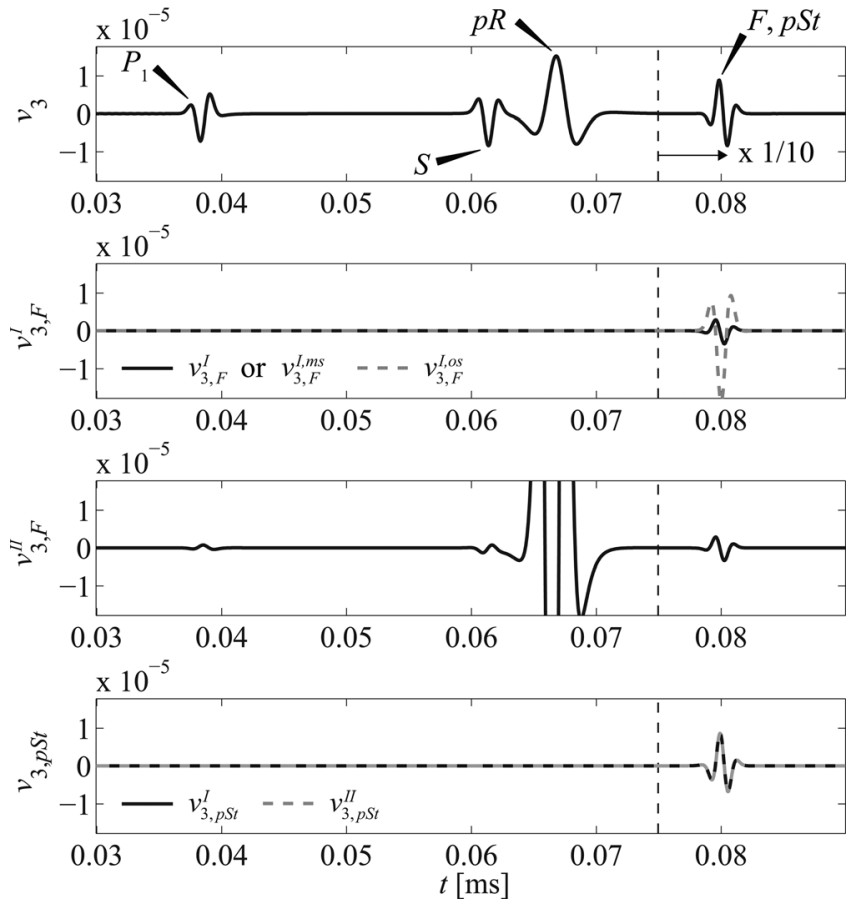

FIG. 9. Bentheimer/light-fluid (configuration 1): Full response $\left(v_{3}\right), q_{F^{-}}$ branch cut contributions $\left(v_{3, F}^{I}\right.$ and $\left.v_{3, F}^{I I}\right)$ and its approximations for method I $\left(v_{3, F}^{I, o s}, v_{3, F}^{I, m s}\right)$, and $p S t$-pole residues for both methods $\left(v_{3, p S t}^{I}, v_{3, p S t}^{I I}\right)$, at $x_{2}=x_{3}=0$, and $x_{1}=0.10 \mathrm{~m}$. In $v_{3, F}^{I I}, p R$-wave part is not entirely visible on scale of this figure. Units are $\left[\mathrm{ms}^{-1}\right]$. From dashed vertical line onwards, responses have been scaled by $\frac{1}{10}$.

Fig. 5 we also show the contribution of the $q_{F}$-branch cut in method II $\left(v_{3, F}^{I I}\right)$. Obviously, it contributes to many waveforms including to that of the $S$-wave.

Next, we consider the $p R$-wave. For configuration 1 (Fig. 5), in both methods there is a $p R$-pole present inside the contour. In method I, where it lies on sheet 3 (see Fig. 2), its residue contribution $v_{p R}^{I}$ coincides with the entire waveform. However, in method II where the pole lies on sheet 1 (see Fig. 4), its residue contribution $v_{3, p R}^{I I}$ is significantly different in magnitude and waveform. The $q_{F}$-branch cut $\left(v_{3, F}^{I I}\right)$ also contributes to the waveform (Fig. 5). Both contributions are very strong but partially cancel each other; together they constitute the $p R$-wave. In Fig. 6 we display the locations of the $p R$-pole as encountered in the two methods. Remarkably, there is a difference in both the real and imaginary parts. This illustrates that the $p R$-pole can lie on different Riemann sheets simultaneously at rather different locations ( $p$-values); in fact, we have here two different $p R$-poles. For configuration 2 (Fig. 7) the $p R$-waveform is again fully captured by the $p R$-pole in method I $\left(v_{3, p R}^{I}\right)$, but in method II there is no $p R$-pole at all present inside the contour and hence, the entire waveform is captured by the loop integrals.

So far, the numerical results confirm the findings in Sec. IV C that, in method I, a separate pseudo interface wave is entirely captured by the residue contribution of the corresponding pole in the far-field interface response. The reason is that the loop integral along a branch cut only contributes to a separate body wave (provided that no singularities are in the vicinity of the branch point). For method II, our results show that a loop integral can contribute to the $p R$-wave, and 
several other wavemodes, as it encounters various singularities along the integration path (see Fig. 4). We argue that the loop integrals do even necessarily contribute to the $p R$ wave because the encountered $p R$-pole and its residue differ from those in method I where the pole residue contains the entire waveform. In some situations the $p R$-wave is entirely captured by the loop integrals when there is no $p R$-pole present inside the integration contour (on principal Riemann sheet).

Finally, we discuss the $p S t$-wave. For configuration 2 (Fig. 7) there is a clear difference between the two methods for the $p S t$-wave: The residue contribution $v_{3, p S t}^{I}$ contains the entire waveform, but in method II the waveform is constituted by the residue contribution $v_{3, p S t}^{I I}$ and the loop integral along the $q_{P 2}$-branch cut ( $\left.v_{3, P 2}^{I I}\right)$. For configuration 3 (Fig. 8), however, in both methods the residue contribution of the $p S t$-pole $\left(v_{3, p S t}^{I}\right.$ and $\left.v_{3, p S t}^{I I}\right)$ contains the entire $p S t$-waveform in the full response. In the former situation (configuration 2) the $q_{P 2}$-branch cut lies close to the real axis [cf. Fig. 4 for small $\left.\operatorname{Im}\left(s_{P 2}\right)\right]$, and the $p S t$-pole lies in between. For increasing frequency the $q_{P 2}$-branch cut comes so close to the real axis that the $p S t$-pole even crosses the branch cut and vanishes from the principal Riemann sheet (see also Sec. V C). ${ }^{9}$ For $f \geq 310 \mathrm{kHz}$ the $p S t$-wave is totally captured by the loop integral. For $f<310 \mathrm{kHz}$ it is fully captured by the pole as the loop integral along the $q_{P 2}$-branch cut does not contribute, even though the pole lies close to the integration path. When the integral is evaluated, however, its contribution turns out to be zero. This is necessarily the case because, contrary to the $p R$-pole (discussed above), the $p S t$-pole is exactly the same pole in both methods (same Riemann sheet and location). The residue contributions are identical. As the residue contribution in method I contains the entire waveform, this should also be the case for method II and, consequently, the contribution of any loop integral to the $p S t$-wave has to be equal to zero for $f<310 \mathrm{kHz}$. Now, it is also clear why in the latter situation (configuration 3 ) the $p S t$-pole contains the entire $p S t$-waveform even for method II: It is because the pole lies on sheet 1 over the entire considered frequency range.

In conclusion, the observations in this section illustrate that in method I all information that belongs to a separate pseudo interface wave is captured by the corresponding pole. Hence, the phase velocity and attenuation can be simply computed from the pole location. In method II, generally, these properties should not be computed using the pole location but from an isolated (separate) waveform in the full response. For the $p S t$-wave, however, in method II one can suffice with the pole location for sufficiently low frequencies so that the pole lies on the principal Riemann sheet.

\section{B. Interfering wavemodes}

Next, we consider the interfering (simultaneously arriving) $F$ - and $p S t$-waves in the response for configuration 1 (Fig. 9). There is a strong interference, which is caused by the small difference in propagation velocities. Mathematically, this results in a small numerical distance [Eq. (37)] when the loop integral of the $q_{F}$-cut is approximated (method
I), as announced in Sec. IV B. In Fig. 9 we show the exact $q_{F}$-branch cut contribution $\left[v_{3, F}^{I}\right.$; Eq. (17)] and the corresponding ordinary $\left(v_{3, F}^{I, o s}\right)$ and modified $\left(v_{3, F}^{I, m s}\right)$ saddle-point approximations [Eqs. (29) and (41), respectively]; $v_{3, F}^{I, m s}$ is indistinguishable from $v_{3, F}^{I}$ and therefore not displayed separately. It can be observed that the error in $v_{3, F}^{I, o s}$ is very significant in waveform and magnitude. In Fig. 10 we compare both the saddle-point approximations and the full numerical solution of the branch-cut contribution $\hat{g}_{F, 3}^{I}$ over the considered frequency band. Obviously, the modified approximation is accurate except for low frequencies where the far-field assumption is not valid (there, the fixed propagation distance $r$ is not much larger than the wavelength).

The important notion here is that the interfering $F$ - and $p S t$-waves (Fig. 9) cannot be considered as separate arrivals. The combined waveform is one arrival that is constituted by the $p S t$-pole residue and the $q_{F}$-branch cut contribution. The correction terms in Eq. (41) that modify the phase propagation slowness of the $q_{F}$-contribution [compared to Eq. (29)] support this idea. Similarly, the pseudo-Rayleigh pole can lie in the vicinity of the shear-wave branch point ${ }^{22}$ and constitute together a combined shear-Rayleigh wave.

In method II the combined $F, p S t$-wave is also constituted by the $q_{F}$-branch cut contribution $\left(v_{3, F}^{I I}\right)$ and the $p S t$ pole residue $\left(v_{3, p S t}^{I I}\right)$; see Fig. 9. The pole residue is identical to that in method I $\left(v_{3, p S t}^{I}\right)$ because in both methods the pole is the same (see Sec. V A). The loop integral along the branch cut, however, also contributes to other waves (see Sec. V A).

For comparison, we consider again the $p S t$-wave in configuration 2 (Fig. 7) as discussed in Sec. V A. The wave does not (strongly) interfere with the $F$-wave and it can be verified that the associated numerical distance (in method I) is larger. However, we observe that, even when both wavemodes are mathematically related to individual parts of the Green's function, it can be difficult to separate the waves in time due

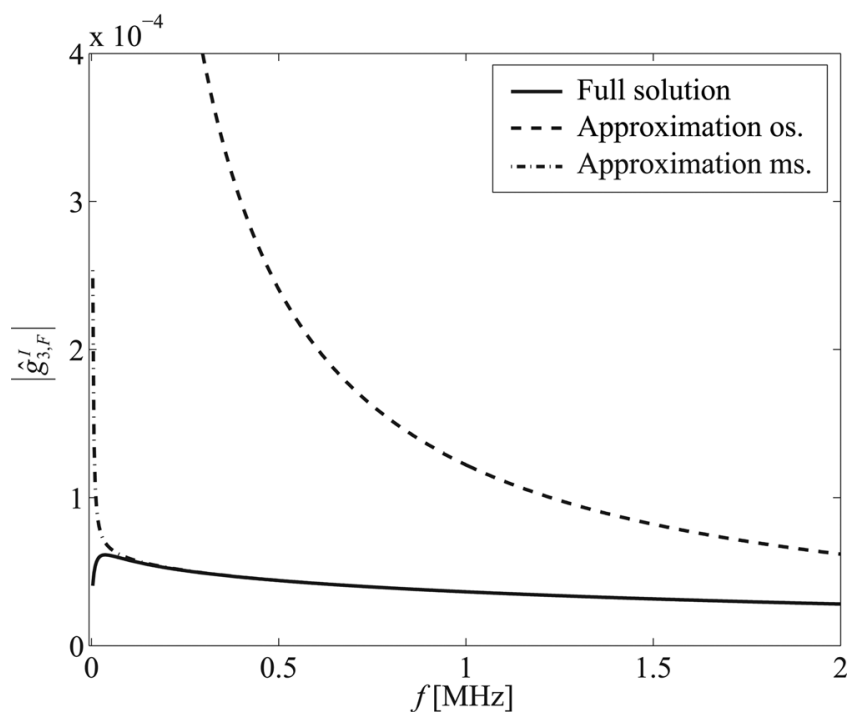

FIG. 10. Bentheimer/light fluid (configuration 1): Full numerical solution of $q_{F}$-branch cut contribution in method I $\left(\hat{g}_{3, F}^{I}\right)$, and its ordinary $\left(\hat{g}_{3, F}^{I, O s}\right)$ and modified $\left(\hat{g}_{3, F}^{I, m s}\right)$ saddle-point approximations, at $x_{2}=x_{3}=0$, and $x_{1}=0.10$ $\mathrm{m}$. Units are $[\mathrm{m} /(\mathrm{Ns})]$. 
to the small difference in propagation velocity and dispersion effects. Recording the waves at very large distances, where the $p S t$-wave has been (fully) separated from the $F$ wave, probably makes no sense because the $p S t$-wave will completely be attenuated due to energy radiation and dissipation. Therefore, one needs to use the entire response or at least the interfering wave-modes for material characterization. Generally, the $p R$-pole and the $p S t$-pole found using the square-root constraints of method I are related to separate arrivals when their real parts are sufficiently different from those of the adjacent branch points.

\section{Migration of poles}

Finally, we discuss the reason of the migration of the pSt-pole from one Riemann sheet to another., ${ }^{8,9}$ Using method II we have found that for configuration 2 the pole vanishes from the principal Riemann sheet at a certain frequency (see Table I). Because the $p S t$-pole found in method I is identical (see Sec. V A), we can use this method to illustrate to which sheet the pole migrates, and explain the physical origin of the phenomenon. We consider the complex $p$ plane (Fig. 2) with the $p S t$-pole lying on sheet 1 . The crucial point is that the branch point of the slow compressional wave comes closer to the real axis when frequency increases. In the limit of $\omega \rightarrow \infty$ it even lies on the real axis because the viscous mechanism is inactivated and the wave propagates without intrinsic attenuation (similar to the $s_{F}$-branch point). However, for increasing frequency the $p S t$-pole does not migrate to the real axis because it continues radiating slow compressional waves (see Fig. 1). Hence, at some frequency the $p S t$-pole must migrate from sheet 1 to sheet 2 as the (transition) line along which $\operatorname{Im}\left(q_{P 2}\right)=0$ migrates toward the real axis. Similarly, the $p R$-pole (found in method I) migrates from sheet 3 to sheet 4 when frequency increases.

In fact, the migration of the poles has already been reported by Gubaidullin et al., ${ }^{7}$ but they did not refer to it as such. They observed that the imaginary part of the square root $q_{\alpha}$ of a radiated body wave can have either positive or negative sign, depending on frequency. Our analysis now provides the physical reason for this.

\section{DISCUSSION}

In the Sec. $\mathrm{V}$ we have shown the transient responses excited at the open-pore interface between a fluid and a porous medium. We would like to emphasize, however, that the general conclusions about the physical interpretation of poles and branch cuts are equally valid for closed-pore conditions or for the boundary conditions in which a finite surface flow impedance is considered. ${ }^{15}$

Closely related to this, it can easily be seen that for a separate "true" interface wave (see Sec. I), ${ }^{2,3}$ in both the methods (I and II) the corresponding pole will contain the entire waveform. This is because the pole lies to the right of every branch point on sheet 1 (see Figs. 2 and 4) at exactly the same location (same pole), where the branch cuts cannot contribute. Therefore, in both methods the pole location provides all information to compute phase velocity and attenuation of the true interface wave.
In our opinion, the general conclusions (from Sec. V) are also not restricted to the configuration of two half-spaces. They capture the situation of a layered rather than a homogeneous porous half-space and the situation where the porous medium includes a third phase (e.g., gas) too. A difficulty will be to find all pole locations accurately. Further, as there will be more interface wavemodes (including higher-order wavemodes), various arrivals can interfere, making physical interpretation of arrivals difficult.

Furthermore, our analysis, which shows that a pseudo interface wave is uniquely described by a pole (method I), could be used as a starting point for the analysis of energy radiation. Gubaidullin et al., ${ }^{7}$ have already addressed this problem, but a more comprehensive analysis is desirable and should include transient responses to show when exactly the waves are radiative and when not.

Finally, we indicate how our work regarding pseudo interface waves at the fluid/porous-medium interface connects to the literature on the $p R$-wave at the fluid/elasticsolid interface. ${ }^{21,29,30}$ Measures for the velocity of the entire $p R$-pulse can be derived based on a zero-crossing in the (exact) time-domain Green's function or on the pulse maximum. ${ }^{29,30}$ These velocity measures differ from the velocity of harmonic waves that can be derived from the location of the $p R$-pole. ${ }^{21,29}$ The latter value represents the phase velocity and is the elastic counterpart of the phase velocity that we have found for the $p R$-pole in method I. In our case, however, this velocity is frequency-dependent and, therefore, it is probably impossible to find similar velocity measures for the entire pulse.

\section{CONCLUSIONS}

The pseudo-Rayleigh $(p R)$ and pseudo-Stoneley $(p S t)$ waves exist at the interface of a fluid and a fluid-saturated porous medium. These pseudo interface waves are related to the $p R$-pole and the $p S t$-pole, respectively, of the poroelastic Stoneley dispersion equation. However, the residue of such a pole does not always capture the entire waveform of the corresponding wave. Therefore, the question arises why and under which conditions the phase velocity and attenuation of a pseudo interface wave can be computed directly from the pole location.

To evaluate the physical interpretation of the poles we computed the point-force response observed at the fluid/ porous-medium interface. We composed the Green's functions using two different methods. In method I vertical branch cuts are used for the involved square roots in the complex slowness plane, implying that the contributing poles are exactly found on Riemann sheets determined by the radiation condition (see Refs. 2, 4, and 7). In method II hyperbolic branch cuts are used, which imply that the contributing poles are found on the "principal" Riemann sheet (see Refs. 6 and 9).

Using saddle-point approximations we showed that, in the case of separated waves in the far field, the loop integral along a branch cut only contributes to a single body wave in method I. Consequently, the far-field waveform of a pseudo interface wave is entirely captured by the residue contribution of the corresponding pole. For method II, we illustrated 
that the loop integral along a branch cut can contribute to several waves as it encounters various singularities along its integration path. The loop integrals necessarily contribute to the $p R$-wave because the $p R$-pole lies on a different Riemann sheet and at a different location compared to the $p R$-pole in method I, where it contains the entire waveform. The loop integrals do not contribute to the $p S t$-wave as long as the $p S t$ pole lies on the principal Riemann sheet because this pole is identical to that in method I, where it captures the entire waveform. At a relatively high frequency, however, the $p S t$ pole migrates to another Riemann sheet due to the slow compressional wave becoming less attenuated; then, the $p S t$-wave is fully captured by the loop integrals in method II.

As a consequence, the phase velocity and attenuation of a separate pseudo interface wave can be simply computed from the location of the corresponding pole in method I. In method II, generally, these properties should be extracted from isolated waveforms in the full response as the pole does not necessarily provide all information to compute phase velocity and attenuation.

\section{ACKNOWLEDGMENTS}

This research is supported by The Netherlands Research Centre for Integrated Solid Earth Sciences (ISES).

${ }^{1}$ J. H. Rosenbaum, "Synthetic microseismograms: Logging in porous formations," Geophysics 39(1), 14-32 (1974).

${ }^{2}$ S. Feng and D.L. Johnson, "High-frequency acoustic properties of a fluid/ porous solid interface. I. New surface mode," J. Acoust. Soc. Am. 74(3), 906-914 (1983).

${ }^{3}$ S. Feng and D. L. Johnson, "High-frequency acoustic properties of a fluid/ porous solid interface. II. The 2D reflection Green's function," J. Acoust. Soc. Am. 74(3), 915-924 (1983).

${ }^{4}$ I. Edelman and K. Wilmanski, "Asymptotic analysis of surface waves at vacuum/porous medium and liquid/porous medium interfaces," Continuum Mech. Thermodyn. 14, 25-44 (2002).

${ }^{5}$ J. F. Allard, M. Henry, C. Glorieux, S. Petillon, and W. Lauriks, "Laserinduced surface modes at an air-porous medium interface," J. Appl. Phys. 93(2), 1298-1304 (2003).

${ }^{6}$ J. F. Allard, M. Henry, C. Glorieux, W. Lauriks, and S. Petillon, "Laserinduced surface modes at water-elastic and poroelastic interfaces," J. Appl. Phys. 95(2), 528-535 (2004).

${ }^{7}$ A. A. Gubaidullin, O. Y. Kuchugurina, D. M. J. Smeulders, and C. J. Wisse, "Frequency-dependent acoustic properties of a fluid/porous solid interface," J. Acoust. Soc. Am. 116(3), 1474-1480 (2004).

${ }^{8}$ B. Albers, "Monochromatic surface waves at the interface between poroelastic and fluid half-spaces," Proc. R. Soc. London Ser. A 462, 701-723 (2006).
${ }^{9}$ K. N. van Dalen, G. G. Drijkoningen, and D. M. J. Smeulders, "On wavemodes at the interface of a fluid and a fluid-saturated poroelastic solid," J. Acoust. Soc. Am. 127(4), 2240-2251 (2010).

${ }^{10} \mathrm{M}$. A. Biot, "Theory of propagation of elastic waves in a fluid-saturated porous solid. I. Low-frequency range," J. Acoust. Soc. Am. 28, 168-178 (1956).

${ }^{11}$ M. A. Biot, "Theory of propagation of elastic waves in a fluid-saturated porous solid. II. Higher frequency range," J. Acoust. Soc. Am. 28, 179191 (1956).

${ }^{12}$ M. A. Biot and D. G. Willis, "The elastic coefficients of the theory of consolidation," J. Appl. Mech. 24, 594-601 (1957).

${ }^{13}$ D. L. Johnson, J. Koplik, and R. Dashen, "Theory of dynamic permeability and tortuosity in fluid-saturated porous-media," J. Fluid Mech. 176, 379402 (1987).

${ }^{14}$ D. M. J. Smeulders, R. L. G. M. Eggels, and M. E. H. van Dongen,, "Dynamic permeability: Reformulation of theory and new experimental and numerical data," J. Fluid Mech. 245, 211-227 (1992).

${ }^{15}$ H. Deresiewicz and R. Skalak, "On uniqueness in dynamic poroelasticity," Bull. Seismol. Soc. Am. 53, 783-788 (1963).

${ }^{16}$ D. R. Burns, "Acoustic waveform logs and the in-situ measurement of permeability - A review," in Geophysical Applications for Geotechnical Investigations, edited by F. L. Paillet and W.T. Saunders (ASTM, Philadelphia, 1990), pp. 74.

${ }^{17}$ K. Aki and P. G. Richards, Quantitative Seismology (Freeman and Co., New York, 1980), pp. 128, 194, 198-199, 244-245.

${ }^{18}$ B. A. Fuchs, B. V. Shabat, and J. Berry, "Functions of a Complex Variable and Some of their Applications (Pergamon Press, Oxford, 1964), pp. 178$187,320-327$.

${ }^{19}$ M. Abramowitz and I. A. Stegun, Handbook of Mathematical Functions (Dover, New York, 1972), pp. 358, 364.

${ }^{20}$ J. G. Harris, Linear Elastic Waves (Cambridge University Press, Cambridge, 2001), pp. 52-55.

${ }^{21}$ R. A. Phinney, "Propagation of leaking interface waves," Bull. Seismol. Soc. Am. 51(4), 527-555 (1961).

${ }^{22}$ L. Tsang, "Time-harmonic solution of the elastic head wave problem incorporating the influence of Rayleigh poles," J. Acoust. Soc. Am. 63(5), 1302-1309 (1978).

${ }^{23}$ L. M. Brekhovskikh, Waves in Layered Media (Academic Press, New York, 1960), pp. 245-255, 261-270.

${ }^{24} \mathrm{H}$. Jeffreys and B. S. Jeffreys, Methods of Mathematical Physics (Cambridge University Press, Cambridge, 1946), pp. 501-505.

${ }^{25}$ J. D. Achenbach, Wave Propagation in Elastic Solids (North-Holland Publishing Company, Amsterdam, 1973), pp. 272.

${ }^{26}$ B. L. van der Waerden, "On the method of saddle points," Appl. Sci. Res. B2, 33-45 (1952).

${ }^{27}$ I. S. Gradshteyn and I. M. Ryzhik, Table of Integrals, Series and Products (Academic Press, London, 1980), pp. 338.

${ }^{28} \mathrm{~N}$. Ricker, "Wavelet contraction, wavelet expansion, and the control of seismic resolution," Geophysics 18(4), 769-792 (1953).

${ }^{29}$ W. L. Roever, T. F. Vining, and E. Strick, "Propagation of elastic wave motion from an impulsive source along a fluid/solid interface," Philos. Trans. R. Soc. London Ser. A 251(1000), 455-523 (1959).

${ }^{30}$ J. H. M. T. van der Hijden, "Quantitative analysis of the pseudo-Rayleigh phenomenon,” J. Acoust. Soc. Am. 75(4), 1041-1047 (1984). 